\section{Cahiers de Narratologie}

Analyse et théorie narratives

$7 \mid 1996$

Mélanges espace \& temps

\title{
La clôture narrativePerspectives théoriques et pratiques textuelles. Les choix esthétiques de François Mauriac
}

\section{Alain Tassel}

\section{OpenEdition}

\section{Journals}

Édition électronique

URL : https://journals.openedition.org/narratologie/11783

DOI : 10.4000/narratologie. 11783

ISSN : 1765-307X

Éditeur

LIRCES

Édition imprimée

Date de publication : 1 janvier 1996

Pagination : 85-99

ISSN : 0993-8516

Référence électronique

Alain Tassel, «La clôture narrativePerspectives théoriques et pratiques textuelles. Les choix esthétiques de François Mauriac », Cahiers de Narratologie [En ligne], 7 | 1996, mis en ligne le 27 avril 2021, consulté le 05 mai 2021. URL : http://journals.openedition.org/narratologie/11783 ; DOI : https://doi.org/10.4000/narratologie.11783

Ce document a été généré automatiquement le 5 mai 2021.

Article L.111-1 du Code de la propriété intellectuelle. 


\title{
La clôture narrativePerspectives théoriques et pratiques textuelles. Les choix esthétiques de François Mauriac
}

\author{
Alain Tassel
}

1 La composition d'une œuvre littéraire accomplit son statut artistique. C'est en fonction des principes qui la gouvernent que «le romancier dispose les éléments de son récit pour obtenir certains effets, d'ordre dramatique, esthétique ou philosophique $»^{1}$

2 La composition du récit repose d'abord sur le programme narratif, c'est-à-dire sur les transformations qui vont faire passer d'un état initial à un état final. Mais composer, ce n'est pas seulement mettre en œuvre une dynamique narrative, ni principalement nouer et dénouer une intrigue, composer c'est construire une cohérence qui installe partout les « connexions ", « les échanges multiples ${ }^{2}$ les ramifications qui font, selon Julien Gracq, la beauté du récit.

3 Les trois phases de construction sont : l'ouverture, le développement et la clôture. Nous allons axer notre étude sur la clôture narrative, cette frontière du récit, ce lieu stratégique que Lukacs appelait la " perspective » "le terminus ad quem", qui, selon lui, «détermine la signification de chaque élément d'une œuvre artistique». Nous proposerons dans un premier temps un essai de synthèse des principaux travaux narratologiques consacrés à ce sujet, avant d'examiner les modalités et les fonctions de la clôture narrative chez François Mauriac. 


\section{A) ESSAI DE SYNTHÈSE SUR LA CLÔTURE NARRATIVE :}

\section{I) D'UNE DÉFINITION À UNE AMORCE DE TAXINOMIE}

4 Tout d'abord, par l'expression "clôture ou clausule narrative » nous désignons la séquence finale d'un texte, une séquence d'étendue variable, de quelques lignes à un chapitre (les termes «explicit/désinit ", symétriques d'incipit, s'appliquent d'ordinaire à la dernière phrase d'un récit). C'est donc un segment narratif pourvu d'une certaine consistance. Philippe Hamon note que le sentiment d'achèvement tient à «la perception de l'interaction en un point du texte particulier de trois paramètres : la fin/ la finition/la finalité.$^{3} \mathrm{La}$ fin marque ici la terminaison du récit, la frontière rendue sensible par la disparition des signes. Cette notion, que l'on pourrait qualifier d'architectonique renvoie à la configuration du livre. La finition ressortit à l'esthétique et désigne la réussite formelle, le «fini » de cet espace textuel. La finalité réfère à la fonction idéologique du texte, à l'expression d'une intentionnalité. Philippe Hamon relève une tendance générale à faire coïncider terminaison et signification. Il rappelle que le schéma position d'un problème/solution de ce problème sous-tend de nombreux textes. La clausule est "particulièrement surdéterminée »: elle porte la marque des conventions, des ritualisations, des pratiques littéraires d'une époque ou d'une école littéraire. Elle est soumise à des contraintes à la fois culturelles et textuelles, puisque certains signaux démarcatifs se présentent comme des constantes liées à un genre littéraire. P. Hamon remarque ainsi que « la ritualisation des procédures de clausules se fait principalement à l'intérieur du genre, le genre pouvant être défini comme un horizon d'attente thématique et formel institutionnalisé ». La clôture romanesque est souvent un point d'embrayage, de mise en phase entre :

5 a) Les différentes isotopies du texte, isotopies sémantiques et phonétiques.

b) Textes et extratextes (la clôture renvoie au genre).

c) Énoncé et énonciation (le narrateur énonciateur signale la fin de son message).

8 La taxinomie suggérée par P. Hamon fournit une série d'oppositions binaires que nous pouvons compléter en fonction, d'une part, de quelques phénomènes textuels repérables (situation, aspects de la clôture) et, d'autre part, des effets de lecture induits par cette ultime séquence narrative. Si ces caractéristiques clausulaires s'opposent par paire, elles peuvent également se combiner, s'associer (au sein d'un même paradigme, ou à la faveur d'un croisement des paradigmes).

PROPOSITIONS POUR UNE TAXINOMIE DES CLÔTURES NARRATIVES

(Nous avons repris et complété le tableau que propose Philippe Hamon dans son article.)

\begin{tabular}{|l|l|}
\hline $\begin{array}{l}\text { Clausule interne (fin de n'importe quelle séquence } \\
\text { interne du texte) }\end{array}$ & $\begin{array}{l}\text { Clausule externe (terminaison } \\
\text { proprement dite du texte) }\end{array}$ \\
\hline Clausule intégrée au texte & $\begin{array}{l}\text { Clausule détachée (épilogue, paragraphe } \\
\text { disjoints de la dernière unité textuelle) }\end{array}$ \\
\hline
\end{tabular}




\begin{tabular}{|c|c|}
\hline $\begin{array}{l}\text { Clausule accentuée (symbolisme accru, intrusion du } \\
\text { narrateur...) C'est, dans la Nouvelle, la «fin-chute » (le } \\
\text { moment de plus forte intensité dramatique), ou «la } \\
\text { pointe ", que Florence Goyet définit comme la } \\
\text { traduction d'une tension antithétique. }\end{array}$ & $\begin{array}{l}\text { Clausule inaccentuée (une pause dans un } \\
\text { processus...) }\end{array}$ \\
\hline $\begin{array}{l}\text { Clausule stéréotypée (conforme à un modèle } \\
\text { identifiable) }\end{array}$ & $\begin{array}{l}\text { Clausule renouvelée (non conforme au } \\
\text { modèle canonique) }\end{array}$ \\
\hline $\begin{array}{l}\text { Clausule directive, univoque, caractérisée par } \\
\text { l'imposition d'un sens }\end{array}$ & $\begin{array}{l}\text { Clausule ouverte, ambivalente, sollicitant } \\
\text { la participation du lecteur à la création } \\
\text { du sens. }\end{array}$ \\
\hline Clausule prévisible, annoncée. & $\begin{array}{l}\text { Clausule moins (pas) prévisible, } \\
\text { surprenante. }\end{array}$ \\
\hline $\begin{array}{l}\text { Clausule non déceptive (entièrement isotope et } \\
\text { redondante par rapport au contexte phonétique / } \\
\text { sémantique précèdent) }\end{array}$ & $\begin{array}{l}\text { Clausule déceptive (remet en cause } \\
\text { l'ensemble du contexte précédent) }\end{array}$ \\
\hline $\begin{array}{l}\text { Clausule fermante (déclenchant une activité mémorielle } \\
\text { de rétroaction chez le lecteur). Elle n'annonce aucun } \\
\text { revirement. }\end{array}$ & $\begin{array}{l}\text { Clausule ouvrante (déclenchant une } \\
\text { activité prospective d'attente chez le } \\
\text { lecteur). Elle suggère des prolongements, } \\
\text { laisse en suspens des possibles narratifs. }\end{array}$ \\
\hline
\end{tabular}

9 Si l'on considère la clôture narrative comme une construction du texte qui programme une activité de lecture, d'interprétation, il est alors possible d'envisager une étude des procédés clausulaires du récit. Il conviendrait de conduire cette étude selon une double perspective, synchronique, pour mesurer les contraintes afférentes au genre, et diachronique, pour apprécier la notion d'usage. Mais pour des raisons bien compréhensibles, nous privilégierons une optique synchronique.

\section{II) DESCRIPTION DES PROCÉDÉS CLAUSULAIRES}

10 Les techniques narratives suscitant un sentiment de conclusion tiennent aussi bien à la configuration du texte, à la disposition des énoncés, qu'aux caractéristiques structurelles, thématiques ou linguistiques.

\section{1) La disjonction de l'énoncé conclusif produite par}

11 a) La mise en page : la clausule peut être mise en relief par un énoncé autonome en position détachée sous la forme d'un épilogue (comme dans La peau de chagrin), ou d'une maxime qui joue le rôle d'un résumé ou d'une conclusion morale. Dans Splendeur et misère des courtisanes, une seule phrase fait office de paragraphe et résume la situation du roman.

b) Par le glissement du temps de la fiction au temps de la narration : l'irruption des indices de l'énonciation romanesque crée un puissant effet conclusif. Ainsi le narrateur de René adopte-t-il le ton neutre du chroniqueur pour authentifier son récit: «On montre encore un rocher où il allait s'asseoir au soleil couchant». Le dernier 
paragraphe de Madame Bovary, qui consacre la réussite insolente d'Homais, est également relaté au présent. Le narrateur se fait le chroniqueur de la petite histoire d'Yonville : «Depuis la mort de Bovary, trois médecins se sont succédé à Yonville sans pouvoir $\mathrm{y}$ réussir, tant $\mathrm{M}$. Homais les a tout de suite battus en brèche. Il fait une clientèle d'enfer ; l'autorité le ménage et l'opinion publique le protège.

Il vient de recevoir la croix d'honneur ».

14 Le temps de l'histoire rejoint celui du narrateur : les événements retenus sont alors contemporains de leur narration. Par ce passage au présent le lecteur est plongé dans la réalité ordinaire de la société contemporaine marquée par le triomphe de l'argent et du cynisme. Le finale entretient savamment la confusion entre roman et chronique, banalise l'histoire, sert remarquablement l'esthétique naturaliste de la tranche de vie.

\section{2) Les particularités structurelles du roman porteuses d'un effet de fermeture}

a) Quand un roman s'organise autour d'une quête, la découverte de l'objet de la quête, l'abandon de la quête ou plus rarement sa pérennisation fourniront une clôture.

b) Si l'action est bâtie sur une enquête, une énigme, la séquence finale sera marquée par la révélation de la vérité d'un mystère, par la résolution d'une énigme posée dans le récit. Elle s'accompagne alors de la diffusion d'un savoir, du dévoilement d'un secret maintenu à travers le récit. Le romancier s'inspire parfois des dénouements dramaturgiques, recourt au "deus ex machina", ou au coup de théâtre.

17 c) L'impression d'achèvement ressortit également à l'aboutissement du programme narratif du sujet: la fin du voyage pour les intrigues centrées sur l'accomplissement d'un trajet (l'arrivée de Léon Delmont à Rome dans La Modification), une importante transformation de la situation du héros pour les romans déroulant la vie d'un personnage, sa maturation ou son accès à une forme de sagesse.

18 d) L'effet de clôture a également partie liée avec la disposition des ultimes séquences narratives. Le quatrième et dernier mouvement des Jeunes filles de Montherlant déroule comme en une strette (partie d'une fugue qui précède la conclusion, et dans laquelle le sujet et la réponse se poursuivent avec des entrées de plus en plus rapprochées) les trois séries narratives afférentes à Solange, Andrée et Thérèse dans l'ordre inverse de leur apparition dans le récit, ce qui aboutit à une fermeture en chiasme particulièrement efficace.

\section{3) Les thématiques conclusives}

19 La clausule est souvent mise en relief par une thématique particulière. La fermeture, fréquente dans le roman réaliste, revêt plusieurs formes (métaphore de l'enceinte, de la clôture murale, du silence). La fermeture de l'espace renforce le sentiment d'achèvement (songeons par exemple au choix de «La maison religieuse » dans La Princesse de Clèves). A la fermeture s'ajoutent le mutisme, la nuit, la retraite (celle de Derville à la fin du Colonel Chabert). et quelques conventions terminales comme le mariage ou la chute (l'épilogue revêt alors l'aspect d'une perte, d'une faillite, d'un échec). L'énoncé souligne sa clôture par des métaphores qui renvoient à la notion de fin, de frontière. $P$. Hamon remarque qu'» il s'agit là du procédé symétrique de celui qui fait, à l'incipit des textes, se multiplier naturellement la thématique du début, du commencement, de l'ouverture, de la nouveauté $»^{4}$. 
Un thème conclusif récurrent dans la littérature contemporaine est celui de la solution par l'art. Cette clôture caractérise par exemple A la recherche du temps perdu, Les Faux monnayeurs, La Nausée. Dans La Nausée, la musique se présente à Roquentin comme un ultime recours pour échapper au sentiment de la vanité de l'existence : faire comme le compositeur et la chanteuse, créer une œuvre, un livre de fiction pour être « sauvé ».

21 D'une manière générale, les clausules centripètes coïncident avec la clôture d'un cycle, la fermeture d'un cercle ou d'une boucle. Elles participent à la création d'un monde clos, cohérent, ordonné. L'effet d'achèvement induit par ce choix thématique n'est pas éloigné de celui lié au procédé du cadrage.

\section{4) Le cadrage}

22 Le souci du cadrage, de la mise en relief des bornes du récit a été analysé notamment par le sémioticien russe Boris Uspenski : «Dans une œuvre littéraire, l'effet de cadre apparaît à chaque fois qu'il y a passage à un point de vue extérieur. » ${ }^{5}$. Boris Uspenski s'intéresse, en particulier, à l'intrusion du narrateur à la fin du récit, à l'émergence de la première personne, à l'adresse au narrataire qu'il situe dans la tradition de l'envoi des ballades médiévales : «La présence de la première personne (le "je" du conteur) en fin de texte peut se comparer à l'autoportrait du peintre à la périphérie du tableau $»^{6}$. L'émergence de la voix narratoriale a été nommée "cadence déclarative» par le sémioticien Philip Stevich. Le narrateur intervient pour signaler la fin de son message (l'ultime chapitre de 20000 lieues sous les mers est intitulé « les dernières paroles du capitaine Nemo»), pour guider le lecteur, analyser l'énoncé ou l'énonciation romanesque. La situation narrative finale coöncide parfois avec une évaluation de l'acte narratif lui-même. Dans la dernière page de Michel Strogoff, le narrateur nous informe du mariage de Stogoff avec Nadia, des nouvelles responsabilités qu'il assume auprès du tsar, puis justifie l'interruption de la progression narrative car «ce n'est pas l'histoire de ses succès, c'est l'histoire de ses épreuves qui méritait d'être racontée ».

Comme le remarque justement Philippe Hamon «A la terminaison du texte, lieu métalinguistique par excellence » se déroule « la thématique de l'écriture, du travail de l'écrivain, de l'effort, de la réflexion sur la distorsion des temporalités écriture/ aventure/lecture, lieu d'interrogation rétrospective ou prospective sur le vouloir-dire, le pouvoir-dire ou le savoir-dire ${ }^{7}$. Ainsi, dans l'épilogue de La Peau de chagrin, un narrateur anonyme élucide la signification des deux personnages féminins, Foedora et Pauline, laisse apparaître la dualité de la femme : «Foedora était hier au Bouffons, elle ira ce soir à l'Opéra, elle est partout, c'est, si vous voulez, la Société » ${ }^{8}$ Foedora est clairement présentée comme le symbole des tentations matérielles, alors que Pauline représente l'idéal de l'artiste, l'inspiration poétique. Il arrive que le narrateur délègue sa compétence herméneutique à un personnage de témoin chargé de proposer un jugement éthique, idéologique ou philosophique sur l'univers décrit dans le récit. A la fin d'Une Vie de Maupassant, la servante Rosalie tempère le pessimisme de Jeanne : « La vie, voyez-vous, ça n'est jamais si bien ni si mauvais qu'on croit $»^{9}$. Dans les dernières lignes du Colonel Chabert, l'avoué Derville assume les fonctions idéologiques et testimoniales dévolues traditionnellement au narrateur. Dans un développement oratoire et empreint d'émotion, il dénonce la cruauté sociale, dresse l'acte d'accusation de la société moderne. D'où le commentaire de Pierre Barbéris : «Au dernières lignes. Derville, c'est Balzac, la conscience qui se dégage comme elle peut des réalités bourgeoises, qui ne change rien au monde, mais qui peut l'écrire $» \cdot{ }^{10}$ Dans le discours de 
ce personnage affleure également la fonction testimoniale axée sur l'attestation. Pour légitimer le récit, il porte un jugement sur la nature des relations qui se tissent entre la diégèse et la réalité sociale : « Toutes les horreurs que les romanciers croient inventer sont toujours au-dessous de la vérité. $»^{11}$ relaté au début du roman ou situé dans l'extratexte. Bel-Ami se conclut sur une réminiscence de Georges Duroy qui se représente les charmes de Madame de Marelle. C'est sur le souvenir d'un épisode de jeunesse que se clôt L'Éducation sentimentale, l'histoire de la Turque, celle d'un premier échec. Les derniers mots, «c'est là ce que nous avons eu de meilleur $»^{12}$ témoignent du désir de remonter aux origines. Cet explicit unifie les destinées de Deslauriers et de Frédéric Moreau par une commune dégradation dans la médiocrité.

\section{5) La fréquence de la symbolisation et de la mystification}

Ces deux phénomènes textuels se manifestent certes à tout endroit du texte, mais il surgissent fréquemment à la clôture du roman réaliste ou naturaliste. Le symbole confère une épaisseur sémantique au texte romanesque, l'enrichit de résonances et de suggestions, incite le lecteur à la réflexion, lui montre que l'action romanesque comme la vie, est chargée de leçons et de significations. Dans Paul et Virginie, les maisons abandonnées figurent la fin de l'idylle. L'atmosphère crépusculaire dépeinte dans les dernières lignes du Père Goriot symbolise la fin misérable du principal protagoniste. Quant au processus de mystification il se manifeste par exemple dans la clausule de Germinal. Les mythes de mort et de vie s'épanouissent et se croisent dans ce roman, où le Voreux et le Moloch, liés au grisou, à l'enfer, s'opposent à l'humanité nouvelle, régénérée. L'explicit renvoie à ce mythe cosmique, à ce «saut dans les étoiles sur le tremplin de l'observation exacte " pour reprendre les termes qu'emploie Zola dans Le Roman expérimental. Il met en valeur la dynamique qui permet aux forces progressistes d'arracher les hommes à la malédiction du déterminisme social: «Des hommes poussaient, une armée noire, vengeresse qui germait lentement dans les sillons, grandissant pour les récoltes du siècle futur, et dont la germination allait faire bientôt éclater la terre $"{ }^{13}{ }^{13}$ La puissante amplification de ce finale assure la mutation du roman en mythe, et l'élargissement de l'aventure individuelle en messianisme collectif.

Au terme de ce panorama des techniques d'écriture mises au service de l'esthétique du récit plein, achevé, clos, il convient de s'interroger sur les modalités de ce que Frédérique Chevillot nomme la réouverture du texte, c'est-à-dire les recommencements que porte en germe la clôture narrative.

\section{III) LA RÉOUVERTURE NARRATIVE}

Cette pratique textuelle privilégiée par de nombreux romanciers modernes, qui réfutent le modèle classique du texte clos, est cependant attestée chez les écrivains réalistes, les auteurs de romans-feuilletons ou de romans d'aventure qui ont souvent exploré la thématique anti-conclusive de l'inconnu, du départ, de la naissance. Le Père Goriot se clôt sur la déclaration théâtrale, grandiloquente de Rastignac et sur sa 
décision d'aller dîner chez Mme de Nucingen : ce discours et ce projet annoncent un nouveau récit. Vendredi ou les limbes du Pacifique s'achève sur le baptême du petit mousse de la Goélette anglaise que Robinson prénomme "Jeudi ». La fin du roman marque la renaissance de Robinson. Soucieux d'inspirer au lecteur un "sentiment prospectif d'attente ", pour reprendre les termes de Philippe Hamon, le narrateur peut mettre l'accent sur le caractère nécessairement récurrent, voire cyclique d'un phénomène, d'une situation décrite, comme dans les dernières lignes de La Peste: « Rieux savait (...) que peut être le jour viendrait, où, pour le malheur et l'enseignement des hommes, la peste réveillerait ses rats et les enverrait mourir dans une cité heureuse ${ }^{14}$

De nombreux autres procédés concourent à la réouverture du texte. Songeons, par exemple, au jeu des temps verbaux dans l'épilogue des Choses de Georges Perec. Ce dernier segment textuel, rédigé au futur invite le lecteur à se représenter les conduites et les gestes des personnages au cours de leur voyage ferroviaire. On pense également à l'intervention du narrateur qui suggère des prolongements possibles en utilisant le lexique de l'ouverture. La nouvelle de Tchékhov, La Dame au petit chien, s'achève sur le mot « commencer » : "(...) le plus compliqué, le plus difficile ne fait que commencer. » Le narrateur nous laisse le soin d'imaginer l'avenir de la liaison d'Anna et de Gourov. La clausule surprenante nous invite parfois à nous reporter au début du récit, à procéder à une rétrolecture. Après avoir lu l'explicit de Sur l'eau. - la découverte du « (...) cadavre d'une vieille femme qui avait une grosse pierre au cou. »-, le lecteur ne croit plus à une hallucination du canotier. Il est ainsi amené à relire la nouvelle et à réinterpréter les signes disséminés tout au long du texte.

\section{B) LES CHOIX ESTHÉTIQUES DE FRANÇOIS MAURIAC ${ }^{15}$}

31 Pour éprouver la validité, la pertinence de ces analyses et de ces concepts, je me suis intéressé à l'œuvre romanesque de François Mauriac. Son abondante production (vingtdeux romans publiés entre 1913 et 1969) se distingue par sa diversité, comme par sa très grande qualité. Le problème proprement littéraire que pose la clôture romanesque a attiré son attention. En effet, dans la préface de ses đuvres complètes, il a marqué sa prédilection pour les récits " bouclés », a regretté l'allure trop heurtée, trop hâtive des Chemins de la Mer (1939) : « Je m'emballe à la fin, mets les bouchées doubles, résume en quelques lignes le destin de Pierre Costadot selon une technique empruntée aux derniers chapitres des livres de la comtesse de Ségur» (III, p. 925). Par ailleurs, les critiques ont souvent reproché à Mauriac de rédiger des dénouements trop brusques de convertir précipitamment ses pêcheurs, d'y laisser entrevoir un peu trop systématiquement l'espoir d'une rédemption chrétienne. José Cabanis vit dans le dernier paragraphe des Chemins de la Mer "une conclusion morale bien inutile». Marcel Arland eut ce mot sévère à propos du Mystère Frontenac : «Les romans de Mauriac sont des démonstrations ». Aussi nous proposons-nous d'éclairer les principales caractéristiques de ces clôtures romanesques. L'agencement de notre étude visera à mettre en lumière les fonctions dévolues à ce segment narratif, selon une progression propre à l'univers mauriacien. 


\section{I) LA RÉOUVERTURE NARRATIVE}

32 La clausule mauriacienne ne conduit pas fréquemment le lecteur à imaginer de nouveaux développements en fonction des possibles narratifs. L'intervention finale du narrateur de L'Enfant chargé de chaînes assure le glissement du temps de la fiction au temps de la narration. Les deux derniers paragraphes de ce roman sont rédigés au présent. Ce temps joue ici le rôle d'un embrayage énonciatif. Le narrateur n'évoque pas seulement des actions contemporaines du moment de l'énonciation. Il annonce «les trahisons possibles» de Jean-Paul Johanet, la «future vengeance» de Marthe. Le narrateur de La Pharisienne intervient pour annoncer une manière de suite au roman. Il crée un effet d'attente, prévoit un prolongement narratif nourri par la liaison de Michèle et de Jean de Mirbel : «Je fus comme happé par le drame de ce couple que je raconterai un jour » (III, p. 880).

A quatre reprises, La Robe prétexte (1914), Le fleuve de feu (1923), Thérèse Desqueyroux (1927), et Ce qui était perdu (1930), la clôture coïncide avec le départ du personnage principal. Chez Mauriac cette situation narrative crée moins un effet de fermeture, qu'elle ne suggère d'autres développements. Le départ du narrateur de $\underline{\mathrm{La}}$ Robe prétexte pour la Bavière est associé à la vente du domaine d'Ousilanne. Disjoint du dernier paragraphe par une série de points de suspension, l'explicit traduit la tentative de libération du personnage, sa rupture avec la province. Thérèse Desqueyroux se conclut aussi sur le départ de Thérèse, sur sa déambulation dans Paris : «Elle farda ses joues et ses lèvres, avec minutie; puis ayant gagné la rue, marcha au hasard» (II, p. 106). Livrée à la ville anonyme, elle se retrouve face à "une armée de piétons ", se hâtant de "traverser la chaussée noire avant que l'ait recouverte la vague des taxis " (II, p. 104). Le décor de la liberté retrouvée est aussi celui des nouvelles menaces. Ce départ s'interpréterait alors moins comme une promesse de libération que d'errance. La clausule de Thérèse Desqueyroux se prête à de nombreuses lectures : elle peut se lire comme le terme d'un processus d'effacement ou de dissolution du personnage (au nonlieu juridique de l'incipit répondrait alors la disparition de Thérèse, projetée dans un lieu indéterminé), ou comme un appel à la vie et à la liberté, comme la proclamation d'une irrépressible aspiration à la vie. Si l'on privilégie cette seconde signification, on peut voir dans le départ du personnage, dans son dessein de communiquer avec des " êtres de chair et de sang ( (II, p. 106) une forme de réouverture narrative préfigurant les deux nouvelles Thérèse chez le docteur (1928), Thérèse à l'hôtel (1933), et surtout La Fin de la nuit (1935), autant d'éclairages du cheminement de Thérèse. Dans le dernier paragraphe de Ce qui était perdu (1930), le narrateur relate le voyage ferroviaire d'Alain et de Tota Forcas. Les plongées dans les pensées d'Alain Forcas permettent d'appréhender son dessein d'échapper à l'attirance incestueuse par la solution religieuse. Ce finale prépare la réapparition d'Alain Forcas sous la figure du prêtre dans Les Anges noirs (1936).

34 Cependant, d'une manière générale, Mauriac recherche rarement de tels effets. Il préfère clore le récit, fixer le destin de ses créatures.

\section{II) L'EFFET DE FERMETURE}

Il est rendu sensible par les clausules qui revêtent l'aspect d'un bilan où s'affirment des vues frappantes sur le destin d'un personnage, sur le sens que prend son action. 
Nettement séparé du reste du chapitre par un blanc typographique, caractérisé par l'irruption du temps de la narration, le dernier paragraphe de Préséances (1921) s'organise autour d'un examen de conscience du narrateur homodiégétique, doublé d'une analyse des personnages. Le narrateur confère à Augustin le rôle d'intercesseur. Il fait allusion au dogme de la communion des saints en nourrissant son discours de formules bibliques, telles «le sel de la terre ", métaphore empruntée à l'Evangile selon Saint-Matthieu (V.13). Le finale laisse poindre une forme d'espoir lié au refus du monde, au désir de solitude.

Cette clôture en forme de bilan resurgit dans Le Désert de l'amour (1925). A la faveur d'un dialogue entre Raymond Courrèges et son père s'esquisse une réhabilitation de la famille, définie comme le meilleur rempart contre les passions : «La famille (...) nous détourne de notre plaie secrète, de notre profonde plaie intérieure » (I, p. 857).

L'impression d'achèvement est également obtenue par le déploiement du thème de la mort. Marqué par une nouvelle irruption du temps de la narration, l'explicit du Sagouin (1951) réfère au suicide de Guillou et de Galéas pour souligner la victoire de la nature sur l'homme, de la mort sur la vie. La Chair et le sang (1920) s'achève sur le suicide d'Edward Dupont-Gunther et sur le pressentiment funeste de Claude, qui repère tous les signes d'une tuerie imminente. La tonalité tragique de ce finale est accordé à un roman dont le titre, une expression empruntée à Saint-Matthieu et à Saint-Paul, désigne tout ce qui en l'homme s'oppose à Dieu.

Soucieux de "boucler» son récit, de donner au lecteur une impression de "fini", Mauriac s'emploie à tisser des liens entre l'ouverture et la clôture, à entretenir ce que Jean Touzot a judicieusement nommé « l'effet de tableau ».

\section{III) L'EFFET DE TABLEAU}

Il procède de l'inscription de correspondances, de systèmes d'échos, de rappels, qui relient la clôture à l'ouverture romanesque et invitent le lecteur à s'y reporter.

La réponse à un problème posé dès l'ouverture, en l'occurrence celui relatif à l'existence du mal dans Génitrix, peut créer cet effet de tableau. Dans les dernières phrases de ce roman s'impose l'image de la Vierge noire, empruntée à la statuaire chrétienne, qui transfigure la servante, la bien-nommée Marie de Lados, et apporte une réponse positive aux interrogations de Fernand Cazenave en lui révélant « au-delà de sa propre dureté, un royaume d'amour et de silence » (I, p. 639).

41 Le roman donne aussi l'impression d'être bouclé lorsque la destinée du personnage forme un cycle, s'oriente dans une direction qui ramène, finalement, à la situation primitive. L'itinéraire d'Elisabeth Gornac, l'héroïne de Destins, épouse ce type de trajectoire. D'une part, le finale de ce roman déroule les souvenirs de ce personnage qui se remémore l'enfance de Bob Lagave. D'autre part, l'explicit, un commentaire du narrateur, parachève la construction de ce cycle : «Elisabeth Gornac redevenait un de ces morts qu'entraîne le courant de la vie» (II, p. 210). Le Temps ne sert plus qu'à reconduire à la situation initiale.

Dans près d'un tiers de cette production romanesque, l'effet de tableau coïncide avec un effet de symétrie. La correspondance des deux frontières du récit est obtenue par la reprise du titre (procédé que les narratologues nomment «l'inclusion») ou de la scène liminaire. L'image-titre du premier roman, L'Enfant chargé de chaînes, resurgit dans les 
deux derniers chapitres. Le titre du livre est d'abord inséré dans le discours du personnage, dans sa prière : « Seigneur, après tant d'efforts et de larmes, pourquoi suisje resté l'enfant chargé de chaînes ? (I, p. 77). Jean-Paul Johanet souligne l'échec de sa tentative de libération. Le jugement final du narrateur confirme l'immobilisme de ce personnage falot et irrésolu qui, après son retour auprès de Marthe, « demeure malgré tout un enfant chargé de chaînes » (I, p. 79).

La reprise du titre s'assortit le plus souvent d'un commentaire explicatif destiné à en élucider la signification. Jacques, le narrateur-personnage du second roman de Mauriac, se réfère à la robe prétexte des jeunes Romains pour marquer la rupture avec l'univers maternel, la fin de l'adolescence. L'inclusion de l'image-titre dans les dernières lignes de ce livre crée un vigoureux effet de contraste avec la clausule du premier roman. L'image-titre forme encore l'explicit de La Fin de la nuit. Il est inséré sous la forme d'une syllepse dans les propos qu'échangent Georges Filhot et Thérèse Desqueyroux :

«Je ne fais rien, j'écoute sonner les-heures. J'attends la fin de la vie.

- Vous voulez dire la fin de la nuit?

(...)

- Oui, mon enfant : la fin de la vie, la fin de la nuit » (III, p. 211).

La syllepse superpose trois significations. Une signification temporelle : l'attente des premiers feux de l'aurore. Une signification existentielle: le terme de la vie. Une signification spirituelle: la dissipation des ténèbres qui enveloppent Thérèse Desqueyroux, menacée par la folie.

L'élucidation du titre occupe un paragraphe entier du finale des Chemins de la mer, rédigé au présent éthique. Le romancier file la métaphore de l'aventure maritime, en nous invitant à découvrir dans le titre du roman une désignation oblique et poétique de l'itinéraire entrepris par quelques personnages en quête d'infini. Dans Le Mystère Frontenac, l'intervention didactique du narrateur lève toute ambiguïté sur la signification du titre : «Le Mystère Frontenac échappait à la destruction, car il était un rayon de l'éternel amour réfracté à travers une race» (IV, p. 673). Cette formule conclusive confère une signification religieuse au titre. La cellule familiale dépasse les déterminismes biologique, psychologique, participe d'une réalité transcendante. On remarquera aussi que dans ce roman la scène finale est symétrique de la scène inaugurale : à l'affection maternelle répond l'affection fraternelle. Le frère aîné prend le relais de la mère auprès d'Yves Frontenac. Cette clôture affirme la permanence et la fermeté des liens familiaux, entonne un véritable hymne à la famille rendu sensible par l'effusion lyrique : «O filiation divine ! Ressemblance avec Dieu ! (II, p. 673).

Enfin, il arrive que se dessine une antithèse entre les deux frontières du roman. L'ouverture et la clôture du Baiser au lépreux s'opposent à la manière des deux pans d'un tableau. Le romancier boucle le récit en inversant l'image inaugurale de «l'homme-larve », du laideron. L'explicit déroule la comparaison de Jean Péloueyre avec un chêne noir, concrétise sa transfiguration. Dans une lettre, François Mauriac a assimilé l'itinéraire de son héros à celui des « lépreux des vies de saints qui prennent la figure du Christ ». ${ }^{16}$

47 Comme on a pu le noter, de nombreux romans mauriaciens s'achèvent sur l'affleurement de la Grâce, sur la présence du mystère au sens théologique du terme. L'écrivain a lui-même mis l'accent sur cette récurrence dans sa postface à Galigaï, publiée en décembre 1951: «La Grâce jaillit encore avarement aux dernières pages du 
Sagouïn. Dans Galigaï, pour pressentir que le destin de mes personnages s'oriente vers Dieu, il faudra attendre la dernière phrase, le dernier mot ».

Et, pourtant, ce « quelqu'un » qu'attend Nicolas Plassac à l'endroit où la route traverse le Lignot, c'est Dieu » (IV, p. 450). Il nous paraît donc opportun d'envisager maintenant ces manifestations de la Grâce ou de l'Espérance, ces échappées vers le Ciel.

\section{IV - LE SIGNE DU SALUT OU LA FONCTION HERMÉNEUTIQUE DE LA CLÔTURE}

L'interprétation religieuse de l'itinéraire du personnage principal, de son évolution est assumée tantôt par le narrateur, tantôt par l'un des protagonistes. Le narrateur du Mal (1924) interprète le renoncement de Fabien Dézaymeries à la vie mondaine et à l'amour comme un moment de résipiscence, comme une forme de conversion. Dans le dernier paragraphe, il s'adresse au lecteur pour orienter, pour infléchir son analyse du personnage en dévoilant le ressort profond qui dicte l'attitude de Fabien : "Qu'il ne soit pas jugé sur cette cruauté apparente : pour le salut de sa maîtresse, il avait refusé dans son cœur le bonheur humain et déjà il était mort au monde» (I, p. 734). Or, la conversion de Fabien est peu vraisemblable, ne s'appuie pas sur un véritable cheminement intérieur. Comme l'écrit Jacques Petit, « par un mouvement de confiance, Mauriac brusque la conversion de son personnage » (I, p. 1315). D'où l'ambiguïté de cette clausule: le retour au refuge maternel contredit l'effort de libération, d'affranchissement de l'éducation reçue, une éducation fermée dont le narrateur instruit le procès tout au long du livre.

Le narrateur-exégète de La Pharisienne relate la transformation morale et spirituelle de Brigitte Pian qui se déprend de ses certitudes, de ses préjugés et se convertit à l'amour. Rédigé pour l'essentiel au présent éthique, l'explicit dégage la signification spirituelle de cette mutation pour en proposer une analyse théologique qui oppose «l'ouverture du cœur aux certitudes de la perfection $»^{17}:$ " Notre père n'attend pas de nous que nous soyons les comptables minutieux de nos propres mérites. Elle savait maintenant que ce n'est pas de mériter qui importe mais d'aimer » (III, p. 881).

Louis, le narrateur-personnage du Nœud de vipères accomplit l'ultime étape d'un cheminement spirituel qui le conduit vers la foi, vers le déchiffrement des signes religieux qui ont parcouru ses rencontres. Son journal s'interrompt au moment où il s'apprête à révéler sa conversion. Ses derniers mots, brutalement interrompus, - « cet amour dont je connais enfin le nom ador.» (II, p. 526) -, laissent au lecteur le «soin d'éclairer le tableau de l'intérieur ", pour reprendre les termes de Jacques Petit. Mais la lettre de Jeanine retire toute ambiguïté à ce finale. Jeanine complète l'aveu de Louis et s'appuie sur deux passages des Évangiles (Saint-Matthieu et Saint-Luc) pour éclairer l'évolution intérieure de son grand-père.

52 La lecture religieuse du parcours accompli par le personnage est parfois délégué à ce que Philippe Hamon appelle "les personnages-anaphores", en l'occurrence les personnages chargés d'interpréter des indices et dotés d'une fonction cohésive. Le finale du Baiser au lépreux situe le drame sentimental de Jean Péloueyre, celui du désir et du dégoût, à un niveau métaphysique et religieux. Le curé de campagne interprète les épreuves que s'impose Jean Péloueyre, son ascétique volonté de renoncement, laquelle transfigure sa souffrance en joie. C'est lui qui donne tout son sens au sacrifice de Jean Péloueyre en le rattachant au thème de la réversibilité des mérites: «ce 
mourant (...) donnait sa vie pour le salut de plusieurs» (I, p. 492). L'intrigue de L'Agneau s'achève sur la mort mystérieuse, - accidentelle ou criminelle -, de Xavier Dartigelongue. L'épilogue de ce roman, nettement détaché du dernier chapitre par la mise en page et par l'emploi du caractère italique, réunit Michèle et Jean de Mirbel. Ces derniers s'interrogent sur les causes de la mort de l'Agneau, se livrent à l'exégèse de ses dernières paroles, rapportent les propos des autres protagonistes (Brigitte Pian, le Curé de Baluzac) qui assument la responsabilité de cette disparition. Ils interprètent finalement son sacrifice à la lumière de l'Évangile selon Saint-Marc, fixent son destin.

Par ailleurs, Mauriac rend sensible l'action de la Grâce par le biais de la figure d'analogie qui, selon Jean Touzot «permet au parti-pris esthétique et à la leçon morale de se confondre au terme de l'histoire $\aleph^{18}$. Dans le finale du Fleuve de feu, la métaphore et la comparaison concourent à la métamorphose de Gisèle de Plailly en madone. « Les cheveux touchés d'une flèche de soleil», "auréolée d'un support angélique », elle «ressemble à la Vierge d'une Assomption» (I, p. 578). La comparaison accentue le dessin, force le trait. Trop subite, la conversion de Gisèle satisfait peu la vraisemblance. On pourra préférer à cet épilogue édifiant la clausule du Baiser au lépreux, où, à la faveur d'un glissement analogique, d'une superposition d'images, Jean Péloueyre est transfiguré en chêne noir et en saint: "Ainsi courut Noémi à travers les brandes, jusqu'à ce qu'épuisé, les souliers lourds de sable, elle dût enserrer un chêne rabougri sous la bure de ses feuilles mortes mais toutes frémissantes d'un souffle de feu, - un chêne noir qui ressemblait à Jean Péloueyre » (I, p. 499). Le chêne de la foi est revêtu de la bure du saint. L'explicit confère une portée métaphysique au drame de la mésentente du couple. La métaphore ignée se greffe sur l'image de l'étreinte posthume pour suggérer l'irradiation de la lumière spirituelle à travers l'espace. Les Chemins de la mer s'achève aussi sur l'image d'un feu intérieur, sur l'évocation de «l'œil de cyclope » qui «brû(le) » en Rose Révolou (III, p. 706). La métaphore mythologique figure l'élan quasi mystique qui entraîne le personnage.

Enfin, les signes du salut affleurent à la faveur d'une écriture symbolique. Dans Le Baiser au lépreux, la scène de l'enlacement de l'arbre par Noémi incarne, au-delà de sa réconciliation posthume avec Jean Péloueyre, la découverte de la voie spirituelle. Le finale du Mystère Frontenac a suscité les réserves de nombreux critiques irrités par sa saturation symbolique. L'Espérance, - comprise comme l'une des trois vertus théologales -, y est représentée par l'image du chêne et du pin. A l'arbre rivé à la terre, symbole du néant, s'oppose l'arbre tendu vers le ciel, ce pin qui aspire à une autre réalité. Nourri par le rêve communautaire, l'explicit du roman baigne dans un halo religieux que diffuse l'image christique de l'assomption. L'épilogue des Anges noirs est bâti autour du rachat de Gabriel Gradère, sauvé par l'action du prêtre Alain Forcas. Le symbole chrétien de la croix naît d'un geste familier (le croisement des mains) et de l'accumulation des signes sacramentels (le crucifix). Il accompagne la conversion de l'assassin arrivé au seuil de la mort. Dans ce dénouement où triomphe la Grâce, où s'inscrit la croyance en la réversibilité des mérites, le symbole est le signe sensible de la Révélation.

Si l'on essaie de saisir l'évolution des clôtures mauriaciennes en fonction d'une perspective diachronique, on observe que la présence de la Grâce se manifeste surtout dans les romans des années 1922-1925 et 1930-1936. Elle se fait plus discrète à partir du Sagouin (1951), où elle est seulement suggérée. La coloration nettement théologique des clôtures rédigées entre 1930 et 1936 trouve son origine dans l'approfondissement et 
le renforcement de la foi, dans ce que l'on a appelé, à tort, sa " conversion ». Aidé par l'abbé Altermann, Mauriac a réussi à surmonter la crise spirituelle qui l'a profondément affecté à la fin des années vingt. C'est « le passage de la tiédeur à la ferveur, du geste formel au don intime, (...) de l'inquiétude à la paix $»^{19}$ qui l'a conduit à insuffler à ses romans, toujours marqués par l'expression de la souffrance et du désir, la force de l'Espérance.

Certes, on peut regretter les développements superflus qui affaiblissent le texte, l'allure parfois démonstrative des clausules nourries de références évangéliques. Mais on admire l'habileté du romancier à solliciter toute une gamme de techniques d'écriture pour servir une théologie du Salut, pour laisser sourdre la circulation de la Grâce, et « (...) montrer que l'Espérance chrétienne peut jaillir au cœur même des fatalités qui écrasent l'homme $»^{20}$.

La prédilection de Mauriac pour la construction en boucle est étroitement liée aux modalités de la distribution des personnages dans le roman, à l'accomplissement d'un cercle qui, après une série de méprises, d'égarements ou d'errances, ramène le héros à Dieu, dès qu'il s'ouvre sur les autres, dès qu'il entre dans « l'ordre de la Charité ».

\section{NOTES}

1. M. Raimond, Le Roman, Paris, Armand Colin, 1988, p. 100.

2. Julien Gracq Lettrines, Paris, 1967 pp. 24-25.

3. P. Hamon "Clausules" Poétique, $\mathrm{n}^{\circ}$ 24, 1975, p. 500.

4. P. Hamon, Ibid., p. 516.

5. Boris Uspenski, « Poétique de la composition », Poétique, n 9, 1972, p. 132.

6. Ibid., p. 131.

7. Philippe Hamon, op. cit., p. 514.

8. Balzac, La Peau de chagrin, Paris, (1ère édit. 1831), Folio Gallimard, 1974, p. 379.

9. Maupassant, Une Vie, (1ère édit.1883), Paris, Folio, Gallimard, 1980, p. 270.

10. Balzac, Le Colonel Chabert. (1ère édit. 1832), Préface commentaires et notes de Pierre Barbéris, Paris, Le livre de poche, 1984, p. 264.

11. Ibid., p. 93.

12. Flaubert L'Éducation sentimentale, (1ère édit. 1869), Paris, Garnier Flammarion, 1964, p. 427.

13. Zola Germinal, (1ère édit. 1885), Paris le livre de poche 1983, p. 503.

14. Albert Camus, La Peste, (1ère édit. 1947), Paris, Folio Gallimard, 1976, p. 279.

15. Toutes les références renvoient aux quatre tomes de l'édition complète des œuvres romanesques de François Mauriac, établie et annotée par Jacques Petit, Bibliothèque de la Pléiade, Paris, Gallimard, 1978 à 1985. Nous indiquerons simplement le numéro du tome et la page concernée.

16. François Mauriac Lettres d'une vie 1904-1969, Paris, Grasset, 1981, p. 114.

17. Jacques Monférier, François Mauriac, Du Noud de vipères à la Pharisienne, Paris, Champion, 1985, p. 164.

18. Jean Touzot, La Planète Mauriac, Paris, Klincksieck, 1985, p. 135.

19. Pierre-Henri Simon, Mauriac, Paris, Seuil, collection « Ecrivains de toujours », 1953, p. 75. 
20. Marie-France Canérot, Mauriac après 1930, le roman dénoué, Paris, Sedes, 1985, p. 164.

\section{AUTEUR}

ALAIN TASSEL

Université de Nice-Sophia Antipolis 